\section{Integrative Medicine \\ International}

\title{
Acupuncture Embedding Complication: Second Report of a Rare Case
}

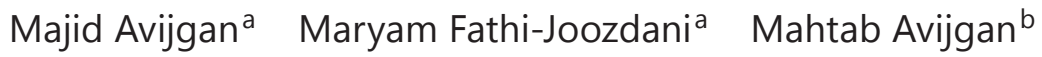 \\ Firoozeh Salehzadeh ${ }^{c}$ \\ anfection Diseases Department, Traditional Medicine Department, Isfahan University of \\ Medical Sciences, Isfahan, Iran; ${ }^{b}$ University of Newcastle, Newcastle, N.S.W., Australia; \\ 'Isfahan University of Medical Sciences, Isfahan, Iran
}

\section{Key Words}

Acupuncture $\cdot$ Aseptic granuloma $\cdot$ Embedding $\cdot$ Vascular headache $\cdot$ Traditional Chinese medicine

\begin{abstract}
Background: In traditional Chinese medicine, one of the modalities of treatment is embedding. Embedding is a method of acupuncture in which catgut is embedded in acupuncture points instead of needles. The current case report describes a rare complication of embedding, and this is the second case report on this modality. Case: A 46-year-old woman suffering from chronic vascular headache (migraine) was referred for acupuncture and embedding. One week after the procedure, she returned with complaints of severe tenderness on the site of embedding. There were tender subcutaneous nodules in the temporal areas of the head. These were diagnosed as suture granuloma, which is one of the rare complications of embedding and has good prognosis. Results: Embedding modality of acupuncture has satisfactory therapeutic effects in many patients but is sometimes accompanied with rare and serious complications. Conclusions: The current authors believe that this complication is a kind of aseptic inflammation and there is no need for any invasive procedure.
\end{abstract}

(C) 2016 The Author(s)

Published by S. Karger AG, Basel

\section{Introduction}

Embedding method has been used in traditional Chinese medicine (TCM) for thousands of years for the treatment of many conditions [1]. Embedding is a method of acupuncture in which catgut is embedded in acupuncture points instead of needles. No complications have 
been reported about this modality with the exception of one case report which was reported recently [2]. The current article introduces a patient with a vascular headache (migraine) who received the traditional embedding acupuncture treatment and in whom a rare complication of procedures occurred.

\section{Case Report}

A 46-year-old woman (housewife) with a 30-year history of chronic vascular headaches (migraines) was referred to acupuncture by a neurologist. The characteristics of the headache were dull heaviness sensation, head distention, accompanied by lightheadedness and headaches that were often retro-orbital, as well as edema and red eyes. The most frequent area of headache was diagnosed in the vertex and less frequently in the frontal area accompanied with episodic neck stiffness. From time to time, she had generalized headaches.

The exacerbation factors were cold weather, fear and anxiety, sexual activity, menstrual period, pre-menstruation period, pressure and palpation, followed by laying down and sleeping. She has a regular menstruation cycle of 4 days bleeding; 2 days bleeding and 2 days spotting, with 22 days interval. Right pulse was palpable in three positions (anterior - middle - posterior), deep, thready, soft and weak, with the same features for left pulse. The tongue was swollen with significant teeth mark and pale with thick white coat.

According to her history and physical examination, her TCM diagnosis was Liver Yang Rising and/or Liver Fire. Due to gastrointestinal symptoms, she also had Damp-phlegm involvement.

The acupuncture points were selected according to Liver disturbance and the following points were selected: GB20; ST8 (crossing point for foot lesser yang and yang linking vessel); CV12 (front mu point of ST which circulates qi in Middle Triple Energizer); LI4 (circulate qi); SP6 (crossing point of Kidney/Liver/Spleen); SP9 (sea point of spleen); Ashi points; GB8 (for dizziness); BL2 and Yin Tang (for frontal headache and runny nose); GV23 (for smell); LU5 (lung qi and phlegm); P6 (for poor appetite); and SP4 (nausea and vomiting by crossing point method); LR13 (stomach fullness).

GB20, ST8, LI4, LIV3, GB8, BL2, Yin Tang as main points and the others as adjacent points were selected.

For each treatment, the main points were selected and based on other symptoms, the other points were added. The points were punctured by needles of 4 or $3 \mathrm{~cm}$ in length and 0.25 in diameter (the brand of needle used is SANCHUANG, China) for $20 \mathrm{~min}$, five times per week.

\section{The Migraine Disability Assessment Test}

The Migraine Disability Assessment (MIDAS) questionnaire is to help measure the impact of headaches on quality of life and provide some information for the primary care provider to determine the level of pain and disability caused by headaches [3-5]. By MIDAS questionnaire, the first four questions are always positive but the fifth question has never been responded and the severity of headache was moderate disability (MIDAS score 11-20).

On the base of TCM differential diagnosis, the Damp-phlegm is the first diagnosis. According to the conceptions and basic sciences of traditional medicine acupuncture, the underlying cause of the headache is mainly a psychogenic base with SP/ST disorder.

\section{Treatment Method}

During the first visit and after ten sessions of treatment, by using the MIDAS scoring system, she declared that the score of her headache had decreased so that the plan of treatment was changed to every other day alternatively. 
Fig. 1. Initial lesion after 1 week.

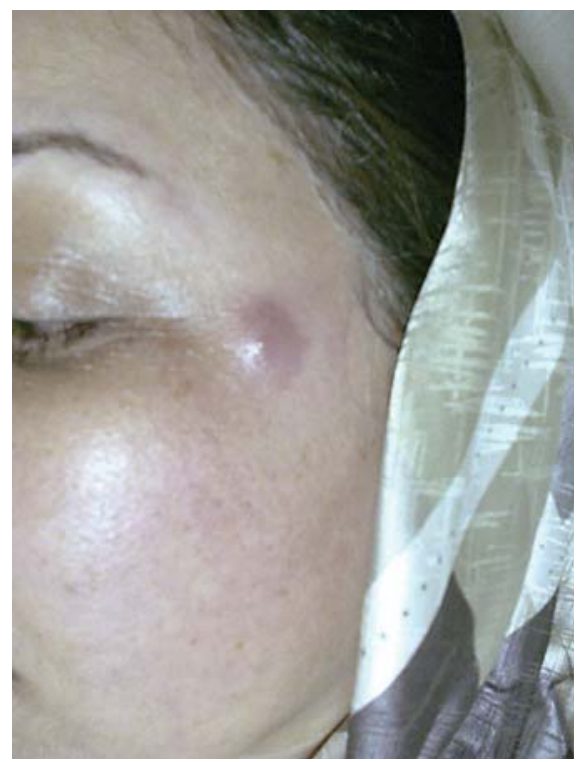

\section{Embedding Method}

The following points were embedded (by $1 \mathrm{~cm}$ of catgut): GB39, EX-HN5, ST25, ST29, ST37 and SP9 [6]. The brand of needle and catgut used is SANCHUANG. The embedding technique was accompanied with a rare complication associated with aseptic inflammation, hence impairing the usual treatment turn. In the assessment of the accompanied lab data for such a complication, there was not any abnormality in the routine laboratory tests, except for a moderate hypersedimentation test (ESR) and a weakly positive CRP.

One week after the procedure, she returned with complaints of severe tenderness, inflammation, and a painful nodule on the EX-HN5 point. There was no fever and no fluctuation (fig. 1). There was tenderness, erythema, and swelling without discharge in the EX-HN5 point, which caused an exacerbation of the headache. The patient declared a feeling of headache around her head, like a circle, while sleeping or laying down. She was advised to use betamethasone ointment topically plus diclofenac suppository for 2 weeks. Since she did not experience a relief in the lesion, it was suggested to consult with a surgeon for excision of the lesion.

There were tender subcutaneous nodules in both temporal areas (fig. 2). During the third week after embedding, the sizes of the lesions were as big as a bean (about $2 \times 1 \mathrm{~cm}$ ). The surgeon suggested nothing except for observation of the lesions. During the fourth week after embedding, one of the lesions became erythematous and the day after that, a fistula appeared in the left EX-HN5 accompanied with getting out a piece of unabsorbed catgut. The thread was extracted by sterile forceps. The other piece of catgut was extracted by pressing on the nodule. In other words, the original catgut was split into 2 parts, each one measuring $0.5 \mathrm{~cm}$ in length (fig. 3).

It is important to note that this patient has experienced a similar reaction called suture granuloma in a suture after a C-section surgery 15 years ago. Suture granuloma is one of the rare complications that happen after surgery, in which a mass of clustered immune cells forms around the area of a surgical incision. It is usually harmless, but can become serious if the cell mass in the granuloma begins to necrotize [7].

Direct smear and culture of discharged material was negative for any bacteria. CBC, sedimentation rate, and CRP were within normal range with no significant abnormality. The pus and discharge of lesion was voided entirely and became subsided within 2 weeks, when only a hyperpigmentation patch was left in that area. The patient used a traditional medicine's 
Fig. 2. The lesion after fistulization.

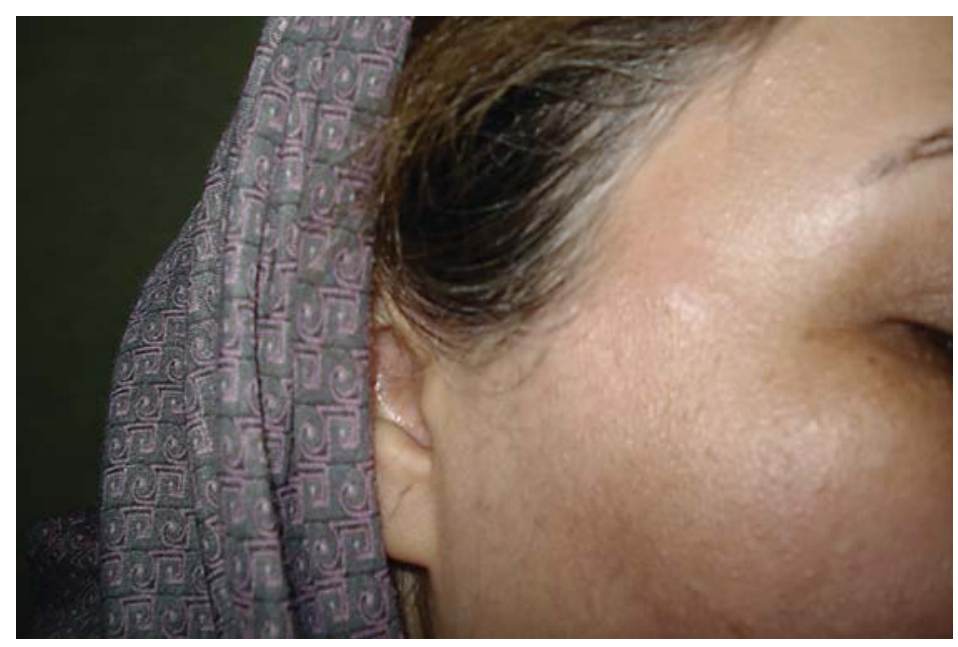

Fig. 3. Catgut divided into 2 parts.

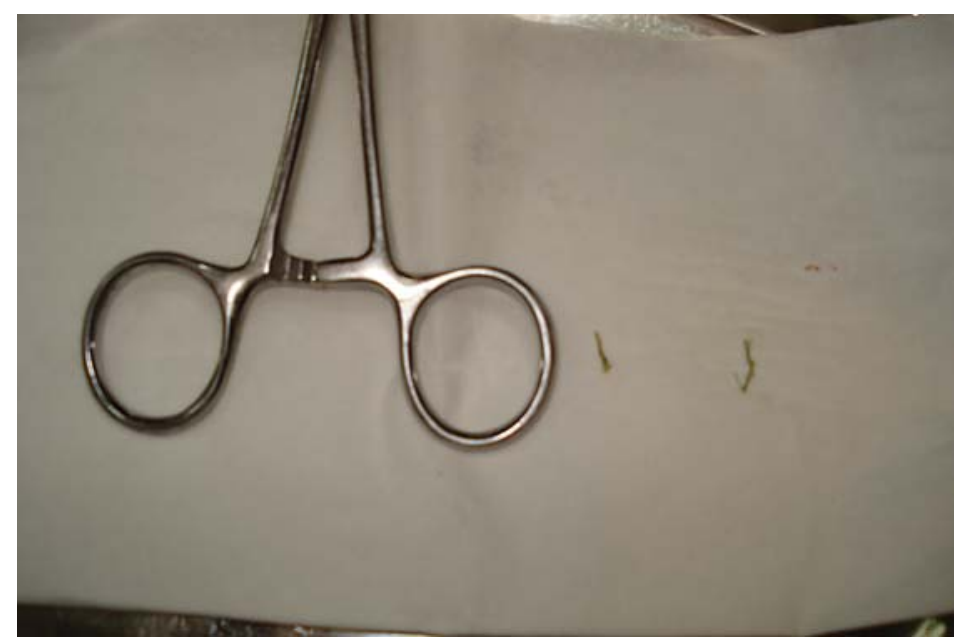

Fig. 4. Lessening after using traditional formula.

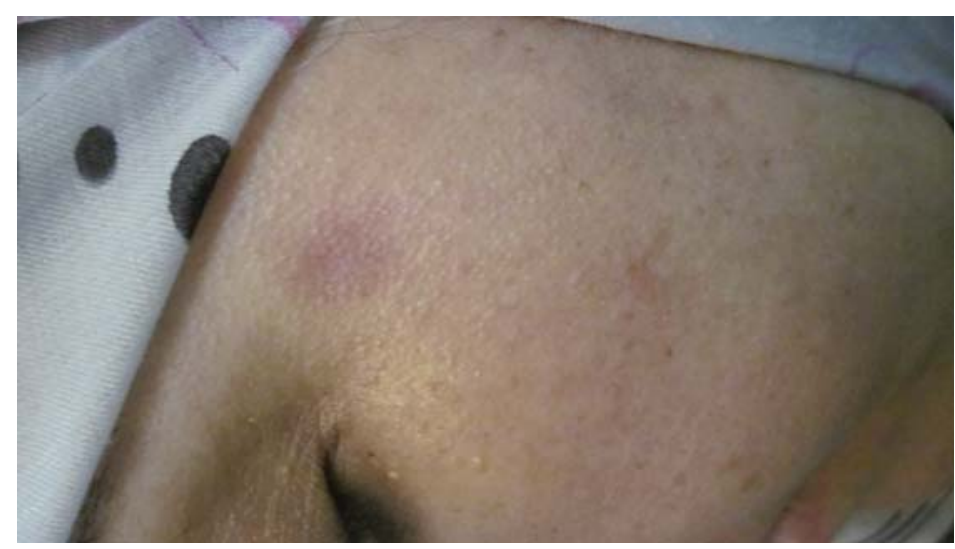

remedy for treatment of the hyperpigmentation for about 1 month, which caused a decrease in the darkness of the hyperpigmented areas (fig. 4).

Four weeks after embedding, the other lesion was still swollen and painful, and by consulting with a plastic surgeon, it was advised to use corticosteroid for 1 month. There were no other suggestions for excision. 
On the right side of the temporal area, during the fourth week, there was an increase in the size of the lesion. Consequently, it was suggested to use corticosteroid (prednisolone) $1 \mathrm{mg} / \mathrm{kg}$ daily. The dosage of prednisolone was tapered during the following weeks, first to $40 \mathrm{mg} /$ day in the second week, then $20 \mathrm{mg} /$ day in the third week, $10 \mathrm{mg} /$ day in the fourth week, and finally down to $5 \mathrm{mg} /$ day. After discontinuing the treatment with corticosteroid (eighth week after embedding), the lesion became suddenly erythematous, tender and swollen again. It seemed that the lesion would have the same prognosis of the left lesion, but 2 days after that, it started regressing and there was a complete recovery afterwards.

During the second month of complications (while using corticosteroids), the patient was suffering from a unilateral headache, which was accompanied with severe morning edema, especially in the periorbital, temporal, superior and inferior eyelid areas. The headaches began as soon as she woke up in the morning, and lasted for 5-6 h. The headache subsided for the rest of the day and began again the next morning. After absorption of the catgut, which lasted for 65 days after embedding, when the signs and symptoms of complication diminished, the severity of the headache was increased. This complication caused an increase in the severity of the pain.

\section{Investigation}

Tissue culture for bacteria, fungus and mycobacterium were performed for this patient and they were all negative.

\section{Outcome}

These tender subcutaneous nodules regressed spontaneously with postinflammatory hyperpigmentation (fig. 1, 2, 4).

\section{Results}

The patient presented with erythematous nodules 2 weeks after embedding, which caused severe tenderness in the area of embedding, as well as an exacerbation of migraine headaches. The patient also reported face swelling and periorbital edema in the morning every day. A general surgeon and also a plastic surgeon were consulted for any probable surgical intervention. The plastic surgeon prescribed a course of prednisone. It was the patient's opinion that, because of the immediacy of the reaction following the embedding, that acupuncture had 'flared' her headache and reaction, and consequently, further acupuncture was discontinued. Additionally, by following up the patient while under prednisone therapy, the signs and symptoms were diminished without any other interventional therapy. Verbal consent was obtained from the patient to undertake her clinical profile for the purposes of this case report.

\section{Discussion}

One modality of TCM is embedding, which is used for the treatment of obesity, premenopausal syndrome, facial paralysis, sciatica, chronic urticaria, ulcerative colitis, depressive neurosis, refractory insomnia, trigeminal neuralgia and Alzheimer disease. Serious complications of embedding are rarely reported [1]. There has only been one case previously published with an embedding complication [2]. The current case report presents a second case with such a rare complication. Similar to the previous report [2], in the pathology of the lesion, there is only an aseptic inflammation, which was not confirmed by any surgical intervention. 
In this article, we are trying to highlight the following points:

- Complications of embedding have rarely happened, but they are possible.

- The complication that happened to the patient presented here is similar to a suture granuloma [7].

- This complication may last a long time, lasting 65 days before complete recovery for the patient presented here.

- There is no need to use antibiotics for the treatment of such a lesion if the embedding was done by a sterile method. Tissue culture and direct smear for bacteria were negative in this patient, similar to the findings in a previous report [2].

- Patients should be reassured that there is no need for any invasive surgery as a treatment for such a complication, especially in a high-risk area like this patient.

- After the regression of the lesion, there may be a hyperpigmentation in the embedding area lasting for several months, as reported in a previous and recent report [2]. Using an herbal formula of traditional Persian-Iranian medicine [8] may decrease the healing period of the hyperpigmentation.

- This experience may show the ability of integrative medicine. Integrative medicine is a combination of treatment, diagnostic method, and management which helps provide a better quality of life with cost benefits and minimum cost [9]. In other words, any method of treatment in traditional medicine and or Western medicine which can help get better may be an integrative medicine.

- We should also ask patients about their history of similar reactions to catgut in their past. The important subject about this complication is a paradoxical phenomenon. In the second month of this complication, we used systemic corticosteroid as a treatment for this lesion, but it did not get better. The lesion flared up after stopping the usage of the corticosteroid and began to regress after a few days.

If the main pathogenesis of this lesion is due to the granuloma formation of the catgut by the immune system, we expect a decrease in the size of granuloma after corticosteroid treatment due to the inhibitor effect on the immune system. Nevertheless, this event did not happen (paradoxical effect). After discontinuation of corticosteroid, we expected a flare-up in the granuloma (that happened) and persistence of this granuloma up to at least 6 months [2]. However, this event did not happen and the granuloma has been absorbed very soon and within 2 days (paradoxical effect).

The current authors suggest that in this case, by corticosteroid therapy, after first inhibition of the immune system and persistent granuloma, the reactivation of immune system by discontinuation of corticosteroid leads to the absorption of the foreign body (catgut) in the granuloma.

More research is needed in the future to investigate whether corticosteroid treatment is recommended for such type of granuloma. This phenomenon needs more immunological discussion concerning the T-cell- and cell-mediated immunity, which is better to be discussed in more detail by future research.

\section{Conclusion}

In this current report, the authors are trying to present a case of a complication after embedding, which is completely relieved without any surgical intervention. However, the course of the underlying disease (migraine headache) and its associated pain deteriorated and the embedding treatment led to an increase in the severity of the pain.

We believe that this complication is a kind of aseptic inflammation and there is no need for any invasive procedures. One should be watching the course of the complication. Based 
on the improvement of the current case, without the use of antibiotic therapy and according to the use of systemic corticosteroid, the current authors conclude that an aberrant reaction is the underlying etiology of this lesion. It is also important to ask patients about the occurrence of a similar reaction to catgut in their past history.

\section{Acknowledgement}

We gratefully acknowledge the patient for her cooperation for this case report.

\section{Disclosure Statement}

No competing financial interests exist.

\section{References}

1 Cheng X: Chapter 14: acupuncture techniques; in: Chinese Acupuncture and Moxibustion, 8th printing. Beijing, Foreign Language Press, 2007, pp 337-361.

2 Chuang YT, Li TS, Lin TY, Hsu CJ: An unusual complication related to acupuncture point catgut embedding treatment of obesity. Acupunct Med 2011;29:307-308.

3 Xu B: Chinese Acupuncture for Weight Loss. Shanghai, Shanghai Scientific and Technical Publishers, 2007, pp 136-139.

4 Garcia-Vivas JM, Galaviz-Hernandez C, Becerril-Chavez F, et al: Acupoint catgut embedding therapy with moxibustion reduces the risk of diabetes in obese women. J Res Med Sci 2014;19:610-616.

5 Li X, Zhang Q, Jiang L, et al: Clinical effect of catgut implantation at acupoints for allergic rhinitis: study protocol for a randomized controlled trial. Trials 2013;14:12.

6 Thirumaran M, Jackson A: Suture granuloma. Postgrad Med J 2004;80:18.

7 Yanagihara M, Fujii T, Wakamatu N, Ishizaki H, Takehara T, Nawate K: Silicon granoluma on the entry points of acupuncture, venipuncture and surgical needles. J Cutan Pathol 2000;27:301-305.

8 Arzani MA: Tebbe Akbari. Persian Medicine (in Persian). Tehran, Institute of Ehyae eIslamic and Iranian Medicine (Tebbe), 2008 (1387 in Persian calendar), pp 1010-1011.

9 Pan W, Zhou H: Inclusion of integrative medicine in clinical practice. Integr Med Int 2014;1:1-4. 